\title{
Comparative genomic profiling of Dutch clinical Bordetella pertussis isolates using DNA microarrays: Identification of genes absent from epidemic strains
}

\author{
Audrey J King*1, Tamara van Gorkom¹, Jeroen LA Pennings², Han GJ van der \\ Heide $^{1}$, Qiushui He ${ }^{3}$, Dimitri Diavatopoulos ${ }^{4}$, Kees Heuvelman ${ }^{1}$, \\ Marjolein van Gent ${ }^{1}$, Karin van Leeuwen ${ }^{1}$ and Frits $\mathrm{R}_{\text {Mooi }}{ }^{1}$
}

Address: ${ }^{1}$ Laboratory for Infectious Diseases and Screening (LIS) Centre for Infectious Disease Control, National Institute for Public Health and the Environment (RIVM), Bilthoven, The Netherlands, ${ }^{2}$ Laboratory for Health Protection Research, National Institute for Public Health and the Environment (RIVM), Bilthoven, The Netherlands, ${ }^{3}$ Pertussis Reference Laboratory, National Public Health Institute, Turku, Finland and

${ }^{4}$ Department of Microbiology and Immunology, University of Melbourne, Victoria, Australia

Email: Audrey J King* - audrey.king@rivm.nl; Tamara van Gorkom - tvangorkom@hetnet.nl; Jeroen LA Pennings - jeroen.pennings@rivm.nl; Han GJ van der Heide - han.van.der.heide@rivm.nl; Qiushui He - qiuhe@utu.fi; Dimitri Diavatopoulos - dimitrid@unimelb.edu.au; Kees Heuvelman - kees.heuvelman@rivm.nl; Marjolein van Gent - marjolein.van.gent@ rivm.nl; Karin van Leeuwen - karinvl@gmail.com; Frits R Mooi - FRmooi@rivm.nl

* Corresponding author

Published: 30 June 2008

BMC Genomics 2008, 9:3।I doi:10.1 |86/I47|-2164-9-3II
Received: 29 January 2008

Accepted: 30 June 2008

This article is available from: http://www.biomedcentral.com/I47I-2164/9/3II

(c) 2008 King et al; licensee BioMed Central Ltd.

This is an Open Access article distributed under the terms of the Creative Commons Attribution License (http://creativecommons.org/licenses/by/2.0), which permits unrestricted use, distribution, and reproduction in any medium, provided the original work is properly cited.

\begin{abstract}
Background: Whooping cough caused by Bordetella pertussis in humans, is re-emerging in many countries despite vaccination. Several studies have shown that significant shifts have occurred in the $B$. pertussis population resulting in antigenic divergence between vaccine strains and circulating strains and suggesting pathogen adaptation. In the Netherlands, the resurgence of pertussis is associated with the rise of $B$. pertussis strains with an altered promoter region for pertussis toxin (ptxP3).

Results: We used Multi-Locus Sequence Typing (MLST), Multiple-Locus Variable Number of Tandem Repeat Analysis (MLVA) and microarray-based comparative genomic hybridization (CGH) to characterize the ptxP3 strains associated with the Dutch epidemic. For $\mathrm{CGH}$ analysis, we developed an oligonucleotide (70-mers) microarray consisting of 3,581 oligonucleotides representing $94 \%$ of the gene repertoire of the B. pertussis strain Tohama I. Nine different MLST profiles and 38 different MLVA types were found in the period 1993 to 2004. Forty-three Dutch clinical isolates were analyzed with $\mathrm{CGH}, 98$ genes were found to be absent in at least one of the B. pertussis strains tested, these genes were clustered in 8 distinct regions of difference.
\end{abstract}

Conclusion: The presented MLST, MLVA and CGH-analysis identified distinctive characteristics of ptxP3 B. pertussis strains -the most prominent of which was a genomic deletion removing about 23,000 bp. We propose a model for the emergence of ptxP3 strains. 


\section{Background}

Bordetella bronchiseptica, Bordetella parapertussis and Bordetella pertussis are closely related respiratory pathogens that infect mammalian species. B. bronchiseptica causes chronic respiratory tract infections in a wide variety of mammals but has only been sporadically isolated from humans $[1,2]$. B. parapertussis consists of two distinct lineages that infect sheep and humans, respectively [3]. B. pertussisis is a human pathogen that causes pertussis, also known as whooping cough, a disease that is particularly severe in infants.

For about 50 years, many countries have been immunizing young children with pertussis vaccines to control disease. Despite vaccination, pertussis remains endemic, with regular epidemic outbreaks. World-wide, whooping cough causes up to 300,000 deaths every year, mostly among unvaccinated infants [4]. Even in countries with a high vaccination coverage, a significant increase in the pertussis incidence has been observed since the 1990s [57]. In the Netherlands, such an increase in pertussis has been observed since 1996 [8].

The resurgence of pertussis in countries with high vaccination coverage has been attributed to a number of factors, including increased awareness with regard to the disease, improved diagnostics, waning vaccine-induced immunity and pathogen adaptation $[4,7]$. Consistent with pathogen adaptation, numerous studies have demonstrated that the $B$. pertussis population has changed in several countries where vaccination has been implemented since the 1950s [9-13]. In particular, antigenic divergence was found between circulating strains and vaccine strains with respect to pertussis toxin (Ptx) and pertactin (Prn).

In the Netherlands this divergence between vaccine and circulating strains has played a role in the reemergence of pertussis $[7,14]$. More recent studies have also found polymorphism in other surface proteins, including tracheal colonization factor A (TcfA) $[15,16]$, the serotype 2 and 3 fimbrial subunits (Fim2 and Fim3) [15,17-19] and in the promoter region for the ptx operon [20]. The latter mutation was found in the Netherlands since the 1990s and we observed that strains with a particular allele of the $p t x$ promoter $(p t x P)$, i.e. ptxP3, have expanded in the Dutch $B$. pertussis population. The increase in frequency of $p t x P 3$ strains coincided with the increase in pertussis notifications in the Netherlands. Moreover B. pertussis strains carrying this novel allele for the pertussis toxin promoter were shown to confer increased virulence [21].

In recent years genomic tools like DNA microarrays have been used for comparison of the genetic composition of different strains within a species. Several microarray-platforms have been developed and applied to specifically address questions related to the genome of $B$. pertussis [2225].

In the study presented here we used Multi-Locus Sequence Typing (MLST), Multiple-Locus Variable Number of Tandem repeat Analysis (MLVA) and a microarray-based Comparative Genomic Hybridization (CGH) method to investigate the heterogeneity of the strains dominating the current Dutch pertussis epidemics, in particularly the ptxP3 strains. We have developed an oligonucleotide microarray representing $94 \%$ of the gene repertoire of the $B$. pertussis Tohama I strain, of which the genome has been sequenced [26]. This microarray allowed us to study the gene contents of the strains involved in the Dutch epidemics and thus to identify (other) polymorphic loci associated with epidemic phenotype. Representative Dutch isolates from 1993 to 2004 were selected since pertussis epidemics have been occurring regularly in the Netherlands since 1996. We aimed to identify MLST, MLVA and gene profiles associated with the Dutch epidemics and to determine the characteristics and heterogeneity of those epidemic strains.

\section{Results}

Dutch strains were selected from the period 1993-2004. The period 1993-1995 was characterized by a relative low level of notifications. In 1996 a sudden increase in notifications was observed and this high level has been maintained until today [5].

\section{MLST analyses}

Three genes known to be polymorphic in the period 1993-2004, ptxP, fim3 and prn, were selected for the MLST analysis of 158 strains. The $p t x P$ gene occurred as two alleles, $p t x P 1$ and $p t x P 3$. The fim 3 gene occurred as four alleles (fim3-1, fim3-2, fim3-3, fim3-4), while three prn alleles were observed (prn1,prn2 and prn3).

We observed 9 different MLST-profiles in the period from 1993 to 2004 (Fig. 1). A number of MLSTs (i.e. MLST141, MLST311, MLST313 and MLST323) were found in low frequencies in the whole period $(<1.5 \%)$ and combined into a single group designated $\mathrm{R}$. The highest frequency of the minor MLSTs (25\%) was found in 1996. For clarity, we will discuss first discuss MLSTs with $p t x P 1$, followed by those harboring $p t x P 3$. Strains with the $p t x P 1$ allele were gradually replaced by $p t x P 3$ strains, which were predominant from 1998 on. The ptxP1 allele was linked to fim3-1 (linkage 100\%) and three prn alleles, prn1, prn2 and prn3 (linkage, respectively, 16\%, 30\% and 54\%). MLST113 predominated until 1996 (frequencies 61\% to 42\%) and showed a gradual decrease in frequency from 1996 to 1999, after which it was not detected. MLST111 was detected in the period 1994-1997 (frequencies 9\%$25 \%)$. MLST112 strains were found in the whole period, 


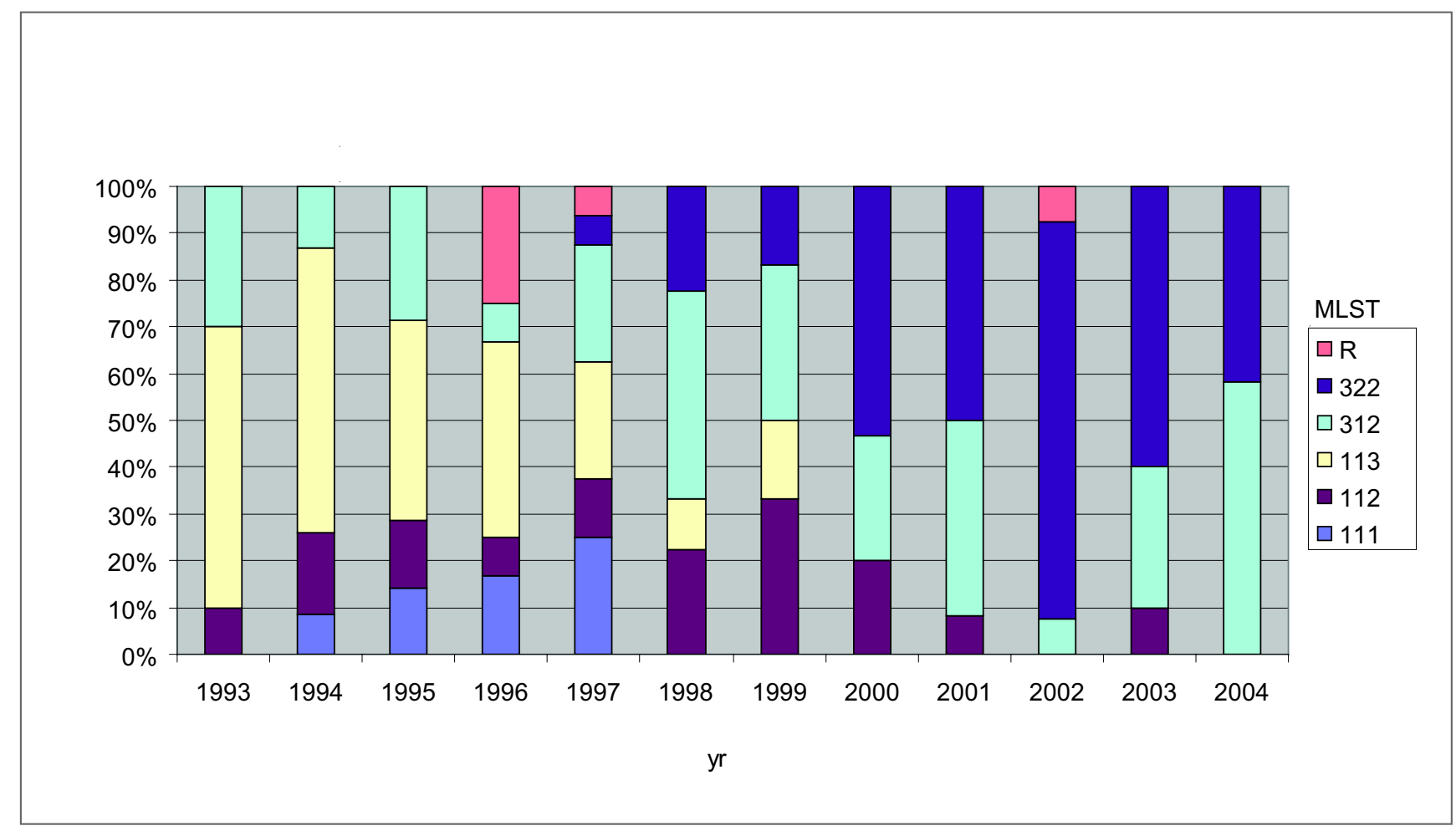

Figure I

Temporal trends in MLST frequencies of Dutch B. pertussis strains from 1993 to 2004. The MLST designation was based on the allele number in the order ptxP, fim3 and prn. E.g. MLSTI I 3 represents strains with ptxPI, fim3-I and prn3. MLSTs found in frequencies less then $1.5 \%$ in the whole period were combined into a single group designated R.

with the exception of the years 2002 and 2004, in frequencies of $8 \%-22 \%$.

In contrast to $p t x P 1, p t x P 3$ was linked to two fim 3 alleles, fim3-1 and fim3-2 (linkage, respectively, 52\% and 48\%). As was observed for $p t x P 1, p t x P 3$ was associated with prn1, prn 2 and prn3 (linkage 2\%, 96\%, 2\%). Thus in contrast to ptxP1, ptxP3 was mostly linked to prn2. Two predominant MLSTs associated with $p t x P 3$ were observed, MLST312 and MLST322. MLST312 was found throughout the whole period and predominated in 1998 and 2004 (frequencies $44 \%$ and $58 \%$, respectively). MLST322 was first detected in 1997 (frequency 6\%) and predominated in the period 2000 to 2003 (frequencies $50 \%$ to $85 \%$ ).

\section{MLVA analyses}

A collection of 222 clinical isolates from the period 1993 to 2004 were typed by MLVA and 38 types were found. Of the 38 MLVA types, 6 have not been described before (see Additional file 1). Most types (64\%) were found in a single isolate only and these were combined into a single group, designated $\mathrm{R}$ (Fig. 2). The remaining four types MLVA26, MLVA27, MLVA29 and MLVA37 were found in frequencies of, respectively, $4 \%, 41 \%, 30 \%$ and $4 \%$ in the whole period. In the period 1993-2004, MLVA29 was found in frequencies of $16 \%-58 \%$, but was not observed in 2001, 2003 and 2004 (Fig. 2). It was the predominant type in the year 1996 (frequency 58\%). MLVA27 was not detected in 1995 and was observed in frequencies of $18 \%$ to $78 \%$ in the other years. It was the predominant type from 1999 to 2004. Compared to MLVA27 and MLVA29, MLVA26 and MLVA37 were detected in much lower frequencies (3\%-21\%), and only in the years 1993-1999. In 1995 the highest frequency of minor types was observed (50\%).

The genetic diversity based on MLVA types showed a decrease from approximately $80 \%$ in the early nineties to 42\% in 2003 and 2004 (Fig. 3 and see Additional file 2).

The relationship between MLST and MLVA types was investigated by constructing a minimum spanning tree based on MLVA-types of 222 B. pertussis strains (Fig. 4). Two main clusters were observed. The majority of the strains in cluster 1 belonged to the related MLVA types 29 and 37. Most of the strains (94.7\%) of MLVA type 29 and 37 had the ptxP1 allele (MLST-profiles 111, 112 or 113). Similarly, most strains in cluster 2 belonged to the related 


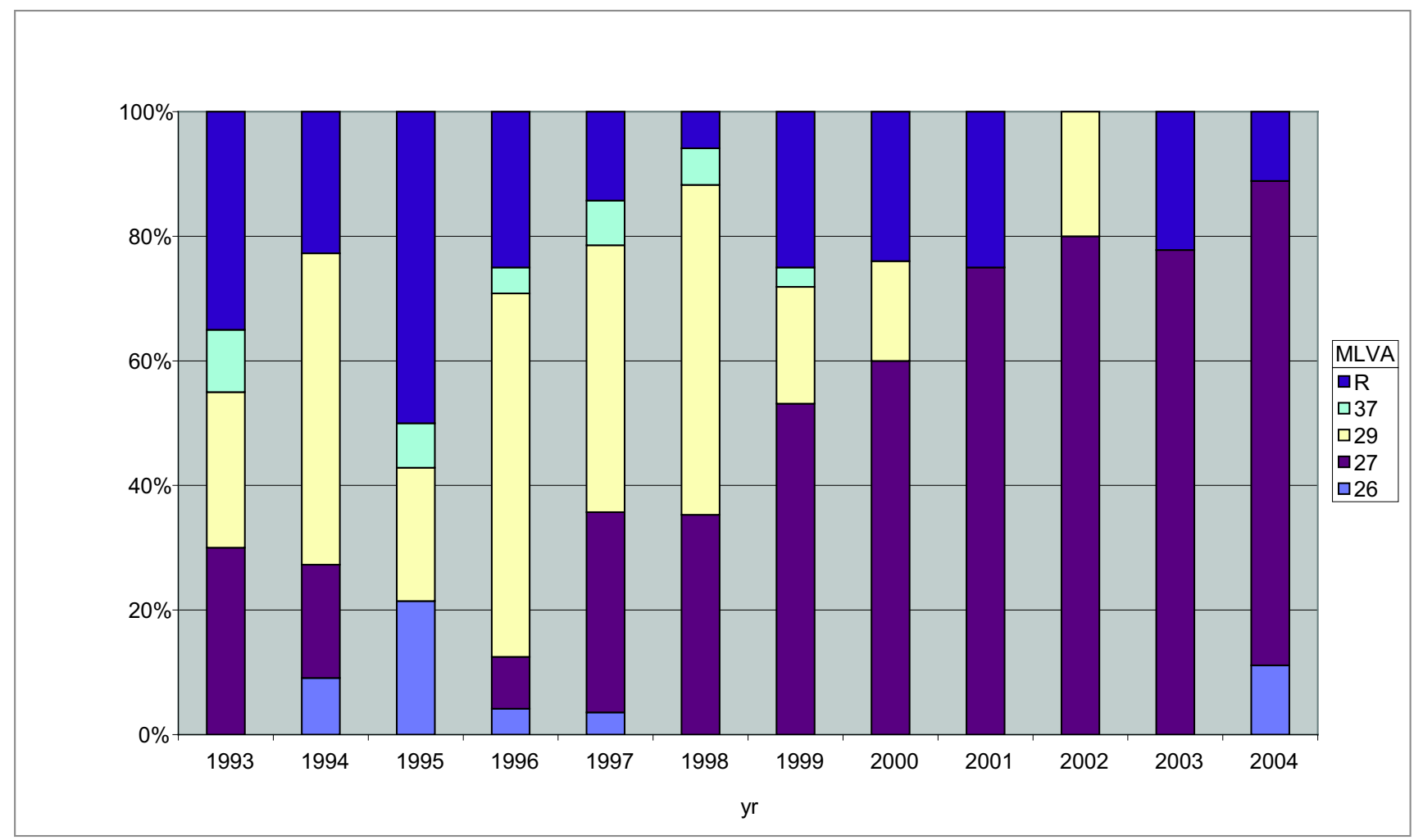

Figure 2

Temporal trends in MLVA frequencies of Dutch B. pertussis strains from 1993 to 2004. Frequencies of different MLVA-types are shown. MLVA types found in frequencies lower than $1.6 \%$ in the whole period were combined into a single group designated $\mathrm{R}$.

MLVA types 27 and 26 and most (92\%) of the strains of these MLVA types carried ptxP3 allele (MLST-profiles 312 or 322).

\section{CGH analyses}

Microarray-based comparative genomic hybridization (CGH) with the $B$. pertussis oligonucleotide microarray based on the Tohama I genome was used for genomic analyses of the ptxP1 and ptxP3 lineages in order to determine if they differed with respect to gene content. Fortythree Dutch clinical isolates, isolated in the period 19932004, were analyzed with CGH, seventeen ptxP1 strains and twenty-six ptxP3 strains. Complete hybridization profiles for twenty-five $B$. pertussis isolates are presented in Fig. 5. Of the 3,581 genes spotted on the microarray, 98 $(2.7 \%)$ were found to be absent in at least one of the $B$. pertussis strains tested. The 98 genes were clustered in eight contiguous loci or regions of difference (RDs) (Table 1).

All strains isolated between 1993 and 2004 contained a deletion in two RDs relative to the Tohama I strain: RD-3 (23 genes, $24.9 \mathrm{~kb}$ ) and RD-5(7 genes, $8.6 \mathrm{~kb})$. The 22 gene products of RD-3 (see Additional file 3 ) were classi- fied in gene class groups (Table 2). Four (17\%) cell envelope proteins, three $(13 \%)$ regulators and $2(9 \%)$ proteins involved in transport and binding were observed. Five $(22 \%)$ proteins were hypothetical proteins, seven (30\%) were proteins with an unclassified function. One gene product $(4.3 \%)$ is involved in central intermediary metabolism and one $(4.3 \%)$ in energy metabolism. Four of the genes $(17 \%)$ were pseudo genes in Tohama I. The RD-5 contained seven genes (see Additional file 4), including three $(43 \%)$ genes, classified in the category transport and binding, coding for proteins involved in iron transport (Table 2). Two genes (29\%) in RD-5 are pseudo genes in Tohama I (Table 2). RD-3 and RD-5 are both flanked by IS481 in Tohama I, on one side and both sides, respectively.

The ptxP1 and ptxP3 lineages were distinguished by RD10 , a region comprising 18 genes and $22.7 \mathrm{~kb}$ (Table 2, and see Additional file 5). RD-10 was present in all ptxP1 strains analyzed. The ptxP3 allele is linked to two fim3 alleles, fim3-1 and fim3-2, and RD-10 is absent in all strains belonging to these lineages. Hierarchical clustering based on CGH data using Pearson correlation grouped the 


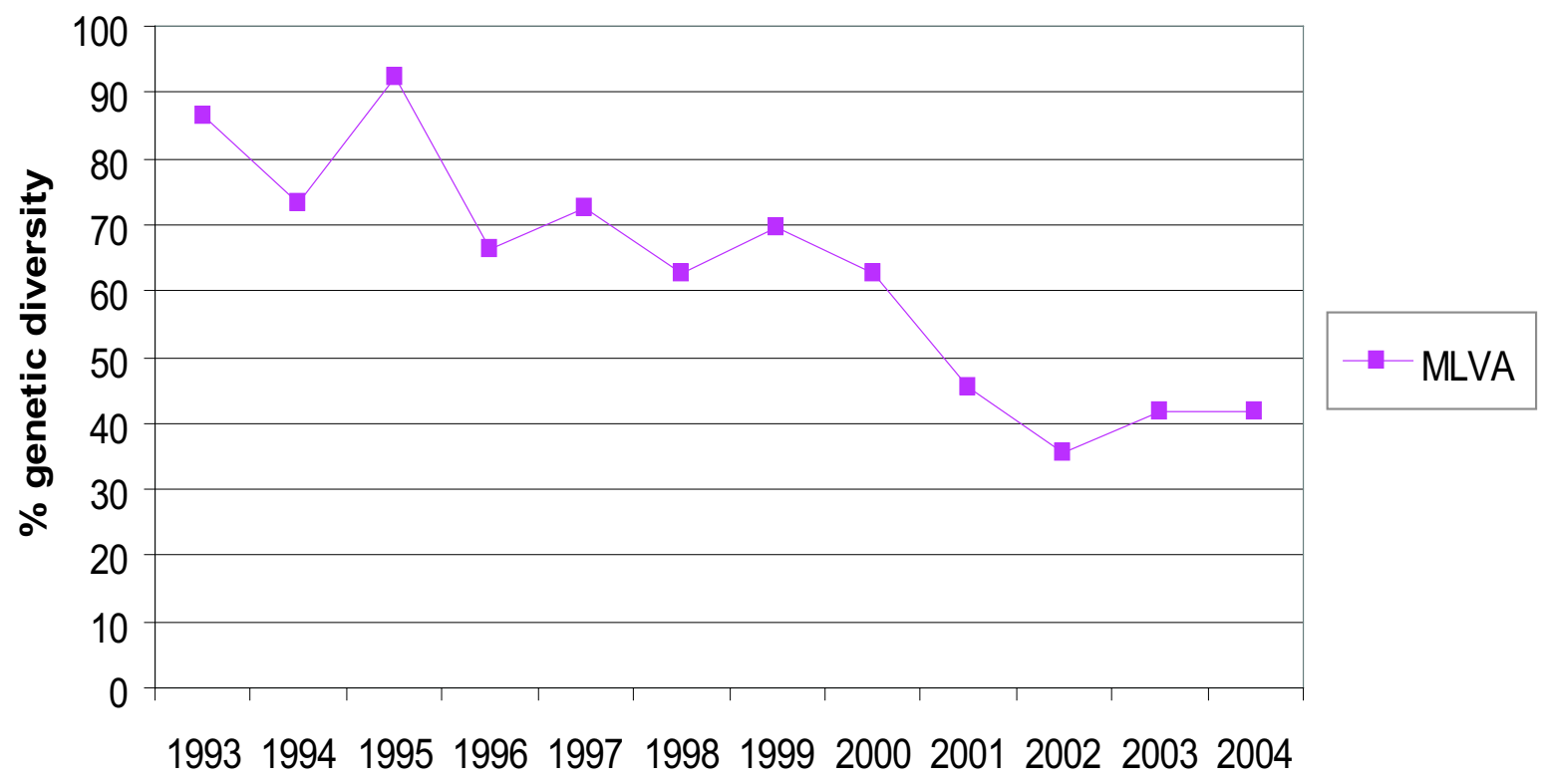

Figure 3

Genetic diversity of the Dutch B. pertussis population in the period 1993 to 2004 . The genetic diversity was calculated for each year using MLVA frequencies.

ptxP1 and the ptxP3 lineages separately (not shown). Of the 18 genes in RD-10 (see Additional file 5), four (21\%) genes were classified in energy metabolism, four $(21 \%)$ genes were involved in transport and binding. Further several single genes $(5 \%)$ coded for a putative exported protein, a transcriptional regulator and a protein involved in iron transport, respectively (Table 2). Five of the 18 genes
(26\%) were pseudo genes in Tohama I. RD-10 is flanked by IS481 elements on both sides in the Tohama I strain.

Four genes present in RD-10 (BP1955-BP1958) were duplicated in the Tohama I genome at positions BP0579BP0582 [26]. In agreement with this, the log ratio values for these genes in strains missing RD-10, were intermedi-

Table I: Presence of regions of difference in Dutch clinical isolates from I993 to 2004

\begin{tabular}{|c|c|c|c|c|c|}
\hline Region of difference Locus: & BP-number & No. of genes & Size (kb) & ptxPI lineage & ptxP3 lineage \\
\hline$R D-3$ & BP0910A-BP0937 & 23 & 24.9 & $0 \%$ (nr strains) & $0 \%$ (nr strains) \\
\hline$R D-5$ & BPI|35-BPI|4| & 7 & 8.6 & $0 \%$ (nr strains) & $0 \%$ (nr strains) \\
\hline$R D-6$ & BPII58-BPII76 & 19 & 18.7 & $94 \%$ (nr strains) & $100 \%$ (nr strains) \\
\hline$R D-27$ & BPI553 & 1 & 0.8 & $82 \%$ (nr strains) & $100 \%$ (nr strains) \\
\hline$R D-10$ & BPI948-BPI 966 & 18 & 22.7 & $100 \%$ (nr strains) & $0 \%$ (nr strains) \\
\hline$R D-28$ & $\mathrm{BP} 2|22-\mathrm{BP} 2| 23$ & 2 & 1.7 & $94 \%$ (nr strains) & $100 \%(\mathrm{nr}$ strains $)$ \\
\hline$R D-29$ & BP2822-BP2839 & 17 & 16.9 & $94 \%$ (nr strains) & $100 \%$ (nr strains) \\
\hline$R D-18$ & ВР33|4-BP3322 & 9 & 9.4 & $94 \%$ (nr strains) & $92 \%$ (nr strains) \\
\hline$R D-I *$ & BP05I5-BP05I6 & 2 & & & \\
\hline
\end{tabular}

Regions of difference (RDs) are numbered according to Brinig et al [23]. RD-I* is part of RD-I as was found by Brinig et al [23]. The percentage of strains harbouring the region and the number of strains analyzed are indicated. Size, refers to the size of the deletion. 


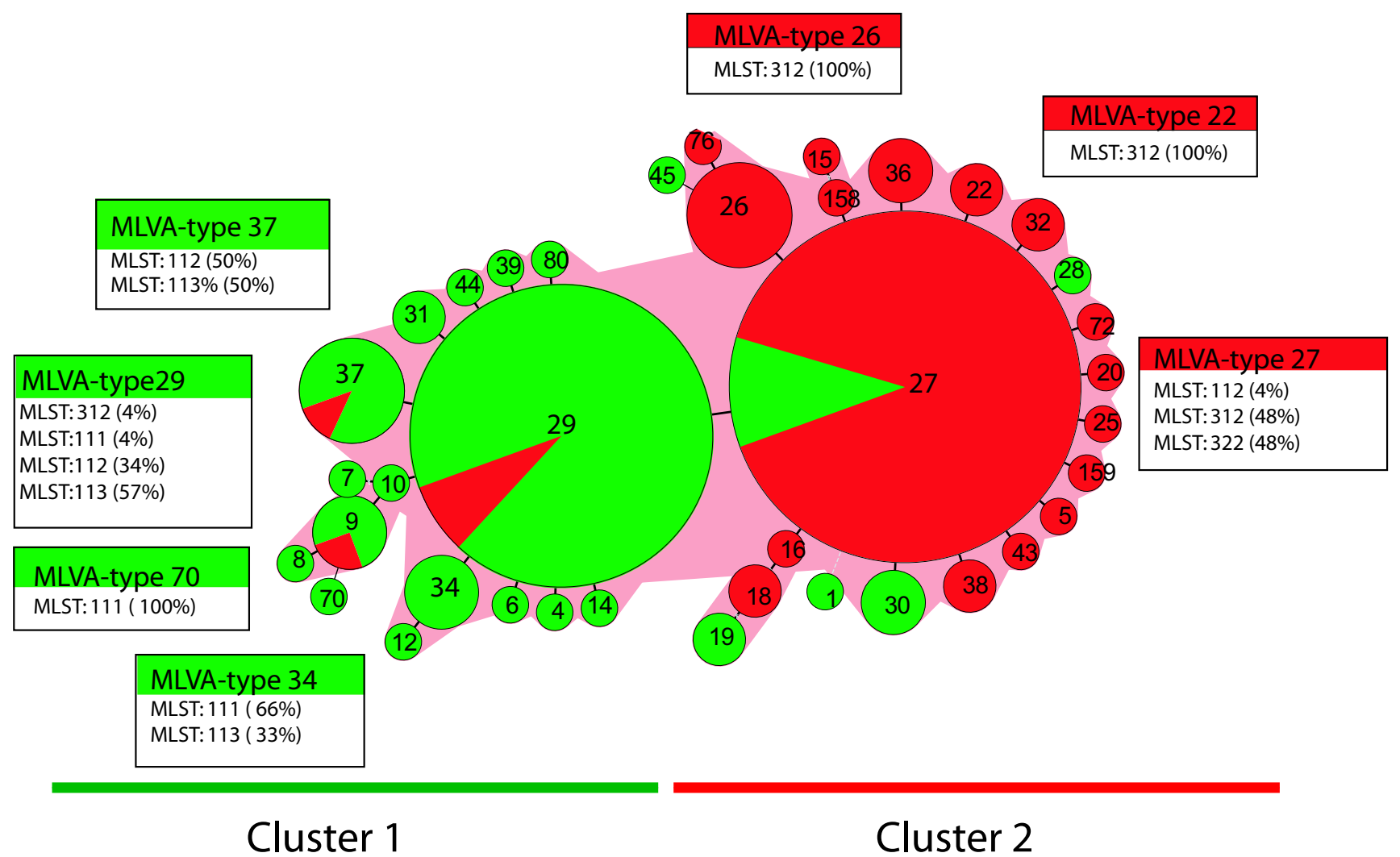

\section{Figure 4}

Minimum spanning tree based on MLVA of Dutch B. pertussis strains isolated from 1993 to 2004. Each circle represents a different MLVA-type, indicated by the number in the middle of the circle. The size of the circle is related to the number of isolates within the particular MLVA-type. Green and red colors indicate, respectively the ptxPI and ptxP3 allele frequencies within MLVA. The distribution of the MLST types is shown in boxes for the most commonly found MLVA types. In some cases the MLST types are not 100\% due to the fact that not all strains were types by MLST.

ate between those for genes which were absent or present as a single copy, respectively. In contrast, identical log ratios were observed between Tohama I and ptxP1 strains indicating that the genes encoded by BP0579-BP0582 are also present at positions BP1955-BP1958 in ptxP1 strains.

B. pertussis strains carrying the ptxP3 allele were isolated for the first time in the Netherlands in 1988. In order to analyze the homogeneity of the ptxP3 strains further, we extended our microarray based CGH analysis to six strains carrying the ptxP3 allele isolated prior to 1993 (data not shown). As was observed for strains from the period 1993 to 2004, RD-10 was missing in all 6 strains. An additional deletion of two genes (RD-1*, BP0515-BP0516) was observed in four of the early ptxP3 strains. The genes in BP0515 and BP0516 encode for a phage-related hypothetical protein and a hypothetical protein respectively (see Additional file 6).
The presence of RD-10 was investigated with PCR in, respectively, 20 and 35 additional $p t x P 1$ and $p t x P 3$ strains. This set of strains included isolates from Denmark, Finland, France, Sweden and the Netherlands. In all cases the absence of RD-10 was linked to the ptxP3 allele.

Interestingly, in strains missing RD-10, the PCR spanning the deletion resulted in two distinct PCR fragments (sizes approximately 1.9 and $2.9 \mathrm{~kb}$ ). DNA sequencing showed that in the $1.9 \mathrm{~kb}$ PCR fragment the genes flanking RD-10, BP1946 and BP1969, were separated by one copy of IS481, while in the $2.9 \mathrm{~kb}$ PCR fragment, two copies of the IS481 separated these genes (data not shown). The second type was found only in strains isolated in 1993-1997.

A number of RDs were found infrequently in 11 strains analyzed with CGH and were not linked to a particular lineage as defined by MLST or MLVA; RD-6 (19 genes, $18.7 \mathrm{~kb}), \mathrm{RD}-27$ (1 gene, $0.8 \mathrm{~kb}), \mathrm{RD}-28$ (2 genes, $1.7 \mathrm{~kb}$ ), RD-29 (17 genes, $16.9 \mathrm{~kb}$ ) and RD-18 (9 genes, $9.4 \mathrm{~kb}$ ) 

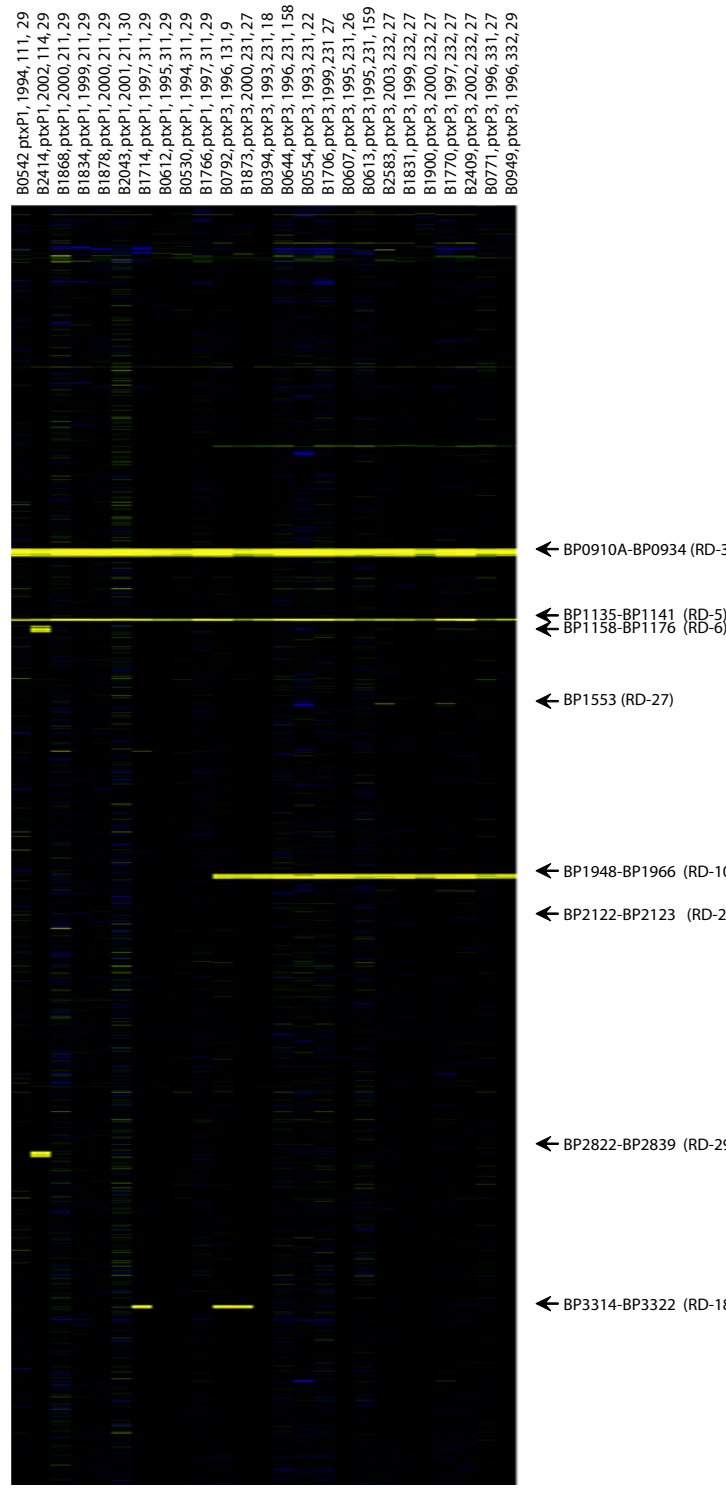

$\leftarrow$ BP2122-BP2123 (RD-28)

$\leftarrow$ BP2822-BP2839 (RD-29)

B

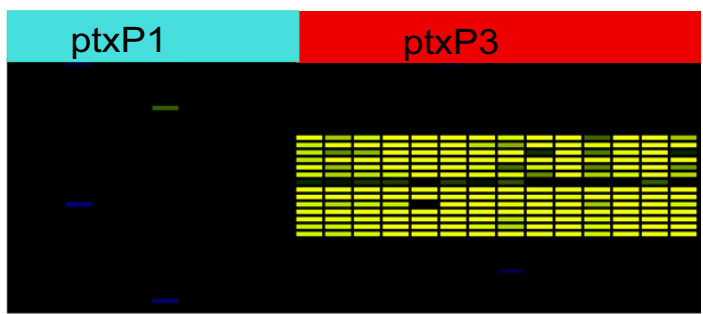

\section{Figure 5}

CGH data for Dutch clinical isolates. CGH analysis of 25 different $B$. pertussis isolates. A. Each column represents one strain. Strain designation, ptxP type, year of isolation, MLST type and MLVA type are indicated above the column. Each row represents one ORF (in B. pertussis Tohama I gene order). Text on the right indicates prominent RD's. B. Gene content of the locus containing BPI948-BPI966 genes (RD-I0) as determined by CGH. Each column represents one strain, strains are sorted by the ptxP type in order to visualize the difference found in this region between ptxPI and ptxP3 type strains and each row represents one ORF (in B. pertussis Tohama I gene order). 
Table 2: Classification of Genes in RDs.

\begin{tabular}{|c|c|c|c|c|c|c|c|c|c|c|c|c|}
\hline Gene class & RD-3 & RD-5 & RD-6 & RD-27 & RD-10 & RD-28 & RD-29 & RD-1 8 & RD-I* & Tot in RD & Tohama I & $\%$ in RD \\
\hline $\begin{array}{l}\text { Amino acid } \\
\text { biosynthesis }\end{array}$ & & & & & & 1 & & & & 1 & 135 & $4 \%$ \\
\hline $\begin{array}{l}\text { Biosynthesis of } \\
\text { cofactors, } \\
\text { prosthetic } \\
\text { groups, and } \\
\text { carriers }\end{array}$ & & & 1 & & 1 & & & & & 2 & 109 & $3 \%$ \\
\hline Cell envelope & 4 & 1 & 3 & & I & & 2 & & & 11 & 505 & $13 \%$ \\
\hline $\begin{array}{l}\text { Cellular } \\
\text { Processes }\end{array}$ & & & 1 & & & & 1 & I & & 3 & 169 & $4 \%$ \\
\hline $\begin{array}{l}\text { Central } \\
\text { Intermediary } \\
\text { metabolism }\end{array}$ & 1 & & 1 & & 1 & & & & & 3 & 174 & $5 \%$ \\
\hline $\begin{array}{l}\text { DNA } \\
\text { Metabolism }\end{array}$ & & & & & & & 2 & & & 2 & 123 & $3 \%$ \\
\hline $\begin{array}{l}\text { Energy } \\
\text { Metabolism }\end{array}$ & I & & 1 & & 4 & & & & & 6 & 429 & $11 \%$ \\
\hline $\begin{array}{l}\text { Hypothetical } \\
\text { proteins }\end{array}$ & 5 & & 2 & 1 & 1 & & 2 & 2 & 2 & 15 & 260 & $5 \%$ \\
\hline $\begin{array}{l}\text { Mobile and } \\
\text { extrachromos } \\
\text { omal element } \\
\text { functions }\end{array}$ & & & & & 1 & & & & & I & 52 & $1 \%$ \\
\hline $\begin{array}{l}\text { Protein } \\
\text { synthesis }\end{array}$ & & 1 & & & & & & & & I & 186 & $5 \%$ \\
\hline $\begin{array}{l}\text { Regulatory } \\
\text { funcions }\end{array}$ & 3 & & 2 & & 1 & & 2 & & & 8 & 322 & $8 \%$ \\
\hline $\begin{array}{l}\text { Transport and } \\
\text { binding }\end{array}$ & 2 & 3 & & & 4 & I & 3 & 1 & & 14 & 327 & $9 \%$ \\
\hline Unclassified & 7 & 2 & 8 & & 5 & & 5 & 5 & & 32 & 940 & $25 \%$ \\
\hline (pseudogenes) & $4(17 \%)$ & $2(28 \%)$ & I (5.2\%) & & $5(26 \%)$ & $2(100 \%)$ & $3(17.6 \%)$ & I (II\%) & & $18(18 \%)$ & 368 & $10 \%$ \\
\hline $\begin{array}{l}\text { Total nr of } \\
\text { genes in RD }\end{array}$ & 23 & 7 & 19 & I & 19 & 2 & 17 & 9 & 2 & 99 & 3731 & \\
\hline
\end{tabular}

The number of genes belonging to COG classes is indicated for each RD, for all RDs combined (Tot in RD) and for Tohama I [26]. In the last column the \% of each class present in all RDs relative to Tohama I is indicated.

(See also Additional files 7, 8, 9, 10 and 11). Sequencing analysis showed that only part $(0.2 \mathrm{~kb})$ of the RD-27 gene was deleted. The five RDs contained 48 genes of which four $(8 \%)$ coded for proteins involved in transport and binding, five $(10 \%)$ for cell envelope proteins and four $(8 \%)$ for regulatory proteins. Seven $(15 \%)$ of these 48 genes are pseudo genes (Table 2). All loci were flanked by IS481 sequences on at least one side in the Tohama I strain. RD-6, RD-28 and RD-29, were each found in a single strain belonging to the $p t x P 1$ lineage. $\mathrm{RD}-27$ was found in 6 strains of the ptxP1 lineage. Finally, RD-18 was found in both the $p t x P 1$ lineage and the $p t x P 3$ lineage in, respectively, 1 and 4 strains. The number of pseudo genes found in the RDs $(18 \%)$ was higher compared to the whole genome $(10 \%)$ [25].

\section{Discussion}

In the Netherlands the upsurge of pertussis was characterized by the expansion of strains carrying a novel allele for the Ptx promoter ( $p t x P 3)$, which completely replaced the resident $p t x P 1$ strains $[20,21]$. It is possible that the ptxP3 allele has increased bacterial fitness and contributed to the upsurge of pertussis. However, the ptxP3 allele may also be linked to other polymorphic loci important for fitness. In this study we have characterized the ptxP1 and ptxP3 lineages using MLVA, MLST and CGH, to establish genetic relationships and to identify loci uniquely associated with $p t x P 3$ strains. Further, this study highlights the microevolution of $B$. pertussis within closely related lineages.

The genetic diversity, based on MLVA frequencies, of the $B$. pertussis population showed a gradual decrease in the period 1993 to 2004 from $80 \%$ to $40 \%$. This reflects the expansion of ptxP3-fim3-2 strains, the population of which was less diverse than the ptxP1-fim3-1 and ptxP3fim3-1 populations (genetic diversities, respectively, 44\%, $63 \%$ and $71 \%$ ). The low genetic diversity of the ptxP3fim3-2 population may reflect recent the expansion of a single clone, consistent with its recent appearance in the Dutch population. 
The emergence of fim3-2 has also been observed in Canada and in Russia $[18,19]$. In view of the many surface antigens expressed by $B$. pertussis, it is remarkable that a single amino acid change in fim 3 may affect the competitive balance between strains. The alleles for the Ptx S1 subunit and Prn code for protein variants which differ in one to seven amino acids $[9,27]$, yet large changes in allele frequencies have been observed over the years, also suggesting that minor mutations may have a noticeable effect on strain fitness.

Consistent with studies from other groups $[23,24,24,25]$, we observed a number for RDs comprising large deletions in $B$. pertussis isolates which were flanked by one or more IS481 copies. A comparison of these studies is complicated by the different designations used for the RDs. We propose a common nomenclature for the RDs observed in $B$. pertussis according to the article by Brinig et al, [23]. In the latter work, 26 RDs were described and we propose that novel RDs should be numbered starting from RD-27. Here RDs were numbered according to Brinig et al [23].

Most frequent were deletions in RD-3, RD-5 and RD-10. RD-3 and RD-5 were absent from all analyzed strains isolated in the period 1993 to 2004 . RD-5 contains several genes that are predicted to be involved in iron uptake, which is essential for survival of most bacteria in the host. However, the $B$. pertussis genome contains several distinct gene clusters involved in iron uptake not all of which may be essential [26]. The absence of RD-10 was associated with ptxP3 strains. RD-10 is comprised of 18 genes, four of which are duplicated and found elsewhere in the Tohama I chromosome: maleate cis-trans isomerase, a probable hydrolase (a pseudo gene in Tohama I), a conserved hypothetical protein and a putative isochorismatase. CGH and PCR analyses indicated that these four genes are duplicated in all strains analyzed belonging to the ptxP1 lineage. The duplication of part of RD-10 may have allowed the preservation of genes important for fitness. Among the $\mathrm{RD}-10$ genes lost in the ptxP3 lineage are those encoding for an $\mathrm{ABC}$ transporter system an iron uptake protein a putative transcriptional regulator and a putative exported protein. The deleted segment did not harbor any known virulence-related genes, nevertheless some of these genes may be involved in virulence. $\mathrm{ABC}$ transporter components can be surface associated and may be involved in virulence [28].

In the $p t x P 3$ strains the region flanking the RD-10 deletion occurred in two forms. In one form the DNA regions flanking the deletion were separated by one IS481 copy and in the second form by two IS481 copies. The form with two IS481 copies was found mostly in strains isolated in 1993-1997 but not in strains isolated after that date. All strains isolated after 1998 were found to have the one copy of IS481 in this region. This suggests that the form with two copies of the IS481 is the precursor form, from which the second form arose after a second recombination step.

A number of RDs were deleted from one to six strains. The RD-18 deletion was observed in two strains characterized by the allele combinations ptxP1-fim3-1 and ptxP3-fim3-2, respectively, and these deletions therefore may reflect independent genetic events, suggesting that deleting RD18 is beneficial for the bacterium.

As yet it is not clear if and how these deletions affect fitness. As observed by others [23-25], we found the deleted regions to be enriched for pseudo genes compared to the genome, suggesting (part of) the deleted regions were not essential. Further, the loss of (functional) genes may increase fitness by lowering metabolic costs and the number of immune targets. In several bacterial species such as Shigella, Chlamydophila, Mycobacterium tuberculosis, Yersinia pestis and Salmonella enterica, the absence of certain genes has been correlated with some beneficial effect such as an increase in pathogenicity or the onset of virulence [29].

\section{Conclusion}

Based on the combined results of the three typing methods, we present a model for the evolution of the ptxP1 and ptxP3 lineages (Fig. 6). It was assumed that point mutations preceded the deletion of RDs. Further variation in Prn was not used to derive relationships between strains, as it is caused by reversible insertion and deletion of repeat units and hence not a useful long-term phylogenic marker [9]. Thus the tree is mainly based on two point mutations found in $p t x P$ and fim 3 resulting in three lineages, ptxP1-fim3-1, ptxP3-fim3-1 and ptxP3-fim3-2. The assumption that variation in Prn occurs at relative high frequency and is reversible, is reflected by the occurrence of all three detected variants in the ptxP1-fim3-1 and ptxP3-fim3-1 lineages (Fig. 6). However, the frequencies clearly differ, Prn3 and Prn2 predominating in, respectively, ptxP1-fim3-1 and ptxP3-fim3-1. Most of the strains in the ptxP3-fim3-2 lineage harbored Prn2 (98\%), while Prn1 was absent (Fig. 6). Clustering based on CGH and MLVA both indicated that $p t x P 1$ and $p t x P 3$ strains represented distinct lineages. The ptxP3-fim3-1 and ptxP3-fim32 strains could not be distinguished by CGH or MLVA, possibly reflecting recent decent from a common ancestor. Since ptxP3 was first detected relatively recently, in 1989 , we presume that this allele was derived from ptxP1, which predominated in the period 1950 to 1989. As fim32 was not detected before 1996, it seems likely that a mutation in a ptxP3-fim3-1 strains resulted in the ptxP3fim3-2 lineage, and this was indicated in Fig. 6. We cannot exclude the alternative hypothesis, however, that the ptxP3 

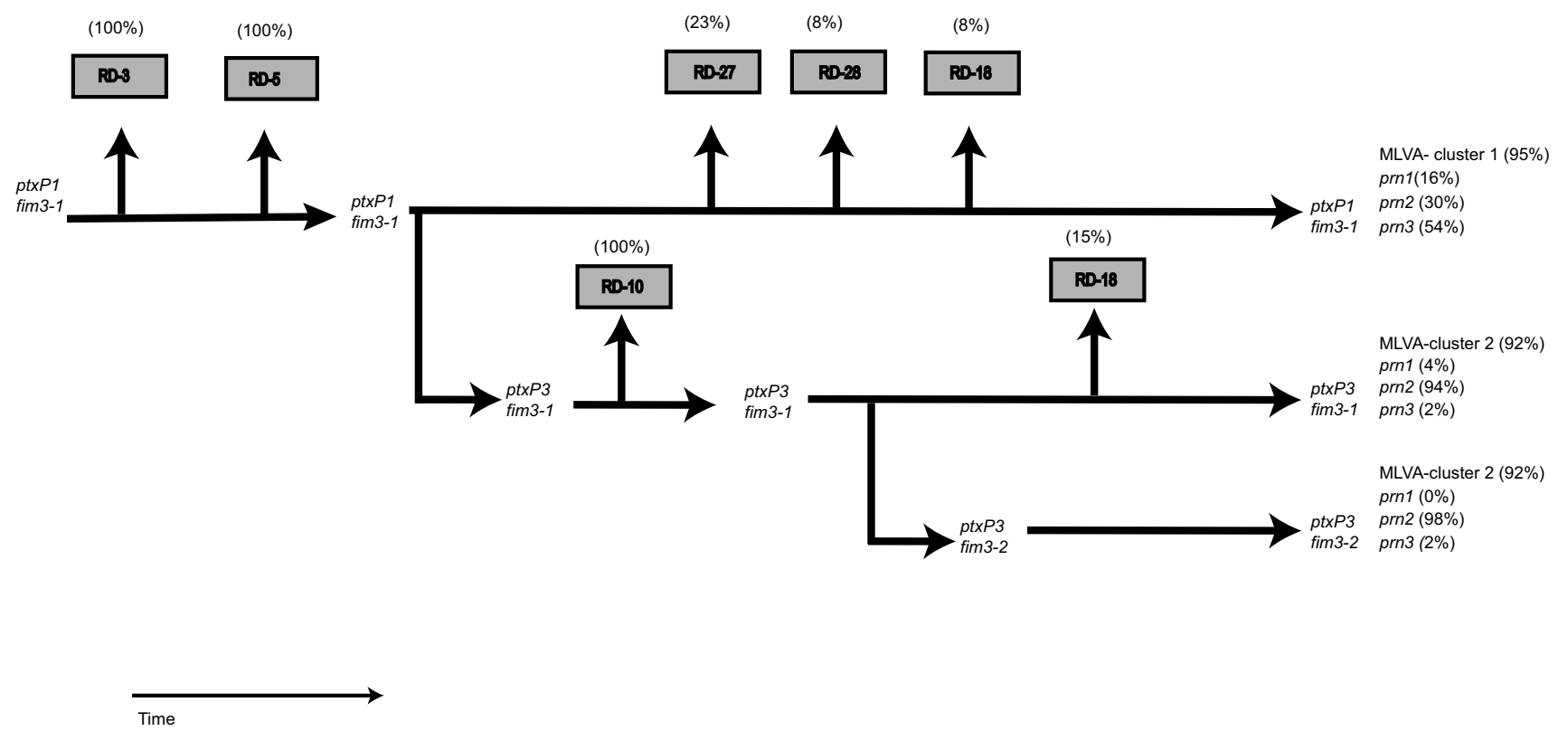

Figure 6

Model for the evolution of the Bordetella pertussis ptxP I and ptxP3 lineages. The tree is mainly based on two point mutations found in $p t x P$ and fim3 resulting in three lineages, $p t x P I-f i m 3-I$, ptxP3-fim3-I and ptxP3-fim3-2. A single strain carrying the $p t x P I-$ fim3-4 alleles was not included. The deleted regions are indicated in boxes and the frequency in the population is shown above the boxes. The frequencies of prn and MLVA types are given in the right part of the figure. See text for further details.

allele arose twice, in a fim3-1 and fim3-2 background. An event which occurred before the branching off of ptxP3, was the deletion of RD-3 and RD-5, two regions which are absent from all the Dutch strains analyzed from the period 1993 to 2004. The absence of RD-10 was characteristic for the ptxP3 lineage suggesting that its deletion preceded the point mutation in $p t x P 3$ or that it occurred early in the history of this lineage (as assumed in Fig. 6). It is also possible that the deletion conferred a strong selective advantage resulting in clonal expansion and replacement of ptxP3 strains still harboring RD-10. An analysis of strains from different European countries by PCR also revealed that $\mathrm{RD}-10$ was absent in ptxP3 strains. Conversely, RD-10 was present in all European $p t x P 1$ strains studied (results not shown).

Brinig et al. [23], also analyzed Dutch ptxP1 strains with CGH and did not observe deletion of RD-10 in these strains. This study did not include any Dutch ptxP3 strains. Strains in which RD-10 was deleted were found in Australia, Italy and the USA [23]. Similarly, Caro et al. [24] and Heikkinen et al. [25] described the loss of RD-10 in B. pertussis isolates in French and Finnish strains (designated $\mathrm{RD}-4$ and locus 3, respectively). In the latter paper the loss of RD-10 was found in all strains of a certain PFGE profile (SR11) that is associated with nationwide epidemics. It would be interesting to determine if in other countries $\mathrm{RD}-10$ loss is linked to ptxP3 as shown in our study.

\section{Methods \\ Strains}

$B$. pertussis isolates were selected from the strain collection of the Dutch National Institute of Public Health. For this study we focused on $B$. pertussis isolates isolated between 1993 and 2004. The selection criteria included the location of isolation and serotype. A total of 222 strains were used for MLVA-typing [20], 133 for MLST typing [9,13], and 43 for CGH analysis (See Additional file 12). The strains selected for CGH analysis were all typed with MLVA and MLST and were representative of the most prevalent MLVA- and MLST-types from 1993 to 2004. This work does not require approval of the ethical commission.

\section{MLVA- typing and MLST-typing}

MLVA typing was essentially performed as described previously in Schouls et al, 2004). For MLST, gene fragments of the pertactin gene ( $p r n)$, the pertussis toxin promoter $(p t x P)$ and the fimbriae 3 gene (fim 3 ) were sequenced essentially as described previously $[15,18,20]$. For the MLST designation the different fim3 alleles were numbered as follows: $f i m 3 A=f i m 3-1, f i m 3 B=f i m 3-2, f i m 3 C=$ 
fim3-3 and fim $3 A^{*}=$ fim 3-4. For each strain, every unique sequence of the ptxP, fim 3 and prn loci received a distinct allele number. The compositions of these loci were expressed in an allelic profile based on the allele numbers. E.g. ptxP3-fim3-1-prn2, was abbreviated to 312. The MLVA and MLST profiles were clustered with the Bionumerics software by using a categorical coefficient and a graphing method called minimum spanning tree [20].

\section{Culture of strains and DNA isolation}

The B. pertussis strains used for microarray analysis in this study are listed in Additional file 12. Strains were cultured for 72 hours on Bordet-Gengou agar plates at $35^{\circ} \mathrm{C}$. Subsequently, they were grown at $35^{\circ} \mathrm{C}$ in Verweij medium (NVI, Netherlands) with $200 \mu \mathrm{g} \mathrm{ml}^{-1}$ heptakis (2,6-di-omethyl)- $\beta$-cyclodextrine for 24 hours while being shaken at $200 \mathrm{rpm}$. Chromosomal DNA was isolated using the Promega Wizard ${ }^{\circledast}$ Genomic DNA Purification Kit (Promega, Madison, USA) according to the manufacturer's instructions. The precipitated DNA was dissolved in $100 \mu \mathrm{l}$ of EB (elution buffer, $10 \mathrm{mM}$ Tris, $\mathrm{pH}$ 8.0) (Qiagen, Hilden, Germany).

\section{B. pertussis DNA array design and construction}

70-mer oligonucleotides were selected from the complete genome sequence of $B$. pertussis strain Tohama I in collaboration with OPERON by using microarray probe design methodology as described by OPERON [30]. Each oligonucleotide was designed with an optimal specificity and sensitivity to its target gene and has an optimized Tm value. A total of 3751 unique 70-mer oligonucleotides, representing $94 \%$ of the genes of a B. pertussis Tohama I strain, were chosen to form the microarray. In addition, 97 oligonucleotides derived from intergenic sequences and 34 control oligonucleotides were included in the microarray. All oligonucleotides were dissolved in 50\% dimethyl sulfoxide and spotted on UltraGAPS coated slides in three replicates (Corning, NY, USA) by ServiceXS (Leiden, Netherlands).

\section{Labeling of genomic DNA}

For each CGH hybridization we labeled the DNA of a test strain and the reference strain ATCC BAA-589 Tohama I. We mixed $4 \mu \mathrm{g}$ chromosomal DNA of $B$. pertussis with 20 $\mu \mathrm{l}$ of the $2.5 \times$ Random Primer Mix (BioPrime DNA labeling kit; Invitrogen) in a total volume of $41 \mu \mathrm{l}$ of water, boiled the mixture for 5 minutes, and then placed it on ice. The samples were centrifuged for 2 minutes at 13,200 rpm and were mixed with $5 \mu \mathrm{l} 10 \times \mathrm{dNTP}$ mix [ $2 \mathrm{mM}$ dATP, dCTP and dGTP, $0.5 \mathrm{mM}$ dTTP (Roche)], $2.5 \mu \mathrm{l}$ of $1 \mathrm{mM}$ Cy3 dUTP (for reference strain) or Cy5 dUTP (for the test strain) (Amersham Biosciences, UK) and $1 \mu \mathrm{l} \mathrm{Kle-}$

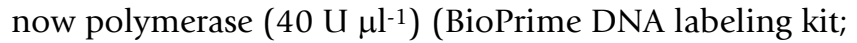
Invitrogen). The samples were incubated for 3 hours at $37^{\circ} \mathrm{C}$. Subsequently, the test and reference samples were purified separately using CyScribe GFX Purification Kit (Amersham Biosciences, UK) and then eluted in $60 \mu \mathrm{l} \mathrm{elu-}$ tion buffer. The incorporation of the $\mathrm{Cy}$ dyes in the labeled target sequences was measured with a NanoDrop spectrophotometer (NanoDrop Technologies). For each $\mathrm{CGH}$ experiment equal amounts of $\mathrm{Cy} 3$ dye (reference) and Cy5 dye (test) were used. The volume of the samples was decreased using a speedvac concentrator (New Brunswick Scientific, Edison, USA). The labeled test and reference samples were mixed together with $79.2 \mu \mathrm{l}$ of hybridization solution [3.44 $\times$ SSC (Invitrogen), $0.32 \%$ SDS (Invitrogen), $1.0 \mathrm{mg}$ yeast $\mathrm{tRNA} / \mathrm{ml}$. Before loading on the microarray the hybridization solution was heated for 3 minutes at $100^{\circ} \mathrm{C}$ and $5.8 \mu \mathrm{l}$ of $10 \times$ DIG blocking buffer was added.

\section{Microarray hybridization}

Before hybridization of the microarrays, $600 \mathrm{~mJ}$ of UV energy was applied to the Corning microarrays using a UV-chamber (Bio-Rad, Richmond, CA, USA) and the microarrays were prehybridized for 45-60 minutes at $42^{\circ} \mathrm{C}$ in pre-warmed prehybridization solution $(5 \times$ SSC, $0.1 \%$ SDS and $0.1 \mathrm{mgml}^{-1} \mathrm{BSA}$ ). The prehybridized microarrays were washed twice for 5 minutes with $0.1 \times$ SSC at room temperature. Finally the microarrays were washed with purified water for 30 seconds and were dried by blowing air with a Quick-Dry-filtered Air Gun (Matrix Technologies Corporation, Hudson, NH, USA). The labeled DNA hybridization mixture was applied to the microarray using lifterslip coverslips and hybridization was carried out in a Genemachines Hybchamber (Genomic Solutions, Ann Arbor, MI, USA) for 14-18 hours at $60^{\circ} \mathrm{C}$ in a water bath. The arrays were then disassembled in $2 \times$ SSC, $0.1 \%$ SDS at $60^{\circ} \mathrm{C}$ and washed for 5 minutes with $2 \times$ SSC, $0.1 \%$ SDS, in a hybridization oven at $60^{\circ} \mathrm{C}$, followed by two washes $0.1 \times$ SSC, $0.1 \%$ SDS for 5 minutes at room temperature and four washes with $0.1 \times$ SSC at room temperature for one minute. Finally the slides were washed for 10 seconds in $0.01 \times$ SSC and then dried using a Quick-Dry-filtered Air Gun (Matrix Technologies Corporation, Hudson, NH, USA). Slides were scanned using a ScanArray 4000XL microarray scanner (Packard BioChip Technologies/Perkin Elmer) equipped with ScanArray express software.

Eighteen and 25 strains analyzed were hybridized at $42^{\circ} \mathrm{C}$ and $60^{\circ} \mathrm{C}$, respectively. Essentially identical results were obtained, but the higher wash temperature resulted in a lower background and these results are shown in Fig. 5. All subsequent washes were performed according to the manufacturer's instruction (Corning, NY, USA).

\section{Image and Data analysis}

The images were analyzed using Genepix Pro 5.1 software (Axon Instruments). Two-color array image data were 
submitted to an in-house microarray database. Raw microarray signal data (no background subtracted) were collected from the database, and after exclusion of control and blank spots the data were normalized in $\mathrm{R}$ [31]. The normalization employed a four-step approach consisting of: (1) $\log 2$-transformation, (2) quantile normalization of all scans, (3) calculating log2 (Cy5/Cy3) ratios per spot, and (4) taking the median from replicate spots. Visualizing the normalized log2-ratios as density plots and quantile-quantile plots showed an approximately normal distribution with an average of 0.012 and a standard deviation of 0.315 . Based on this normal distribution, setting a cutoff for deletion at -1 would predict deleted genes with a False Discovery Rate (FDR, [32]) of $7.7 \%$; setting the cutoff at -1.5 corresponds to a FDR of $0.014 \%$. On the basis of preliminary PCR validations, we determined a cutoff for the normalized intensity ratio of $<-1.5$, indicating the absence of the gene. These genes were selected for further analysis with PCR or sequence analysis. The combined normalized data were visualized with TIGR MultiExperimentViewer [33]. The logarithm of the hybridization ratio $[\log 2(\mathrm{Cy} 5 / \mathrm{Cy} 3)]$ is indicated in the yellow-black-blue color scale. $[\log 2(\mathrm{Cy} 5 / \mathrm{Cy} 3)]=-3=$ Yellow, $[\log 2(\mathrm{Cy} 5 / \mathrm{Cy} 3)]=0$ = Black, $[\log 2(\mathrm{Cy} 5 / \mathrm{Cy} 3)]=+3$ $=$ Blue.

\section{PCR confirmation and sequence analysis}

PCR analysis was employed in order to confirm the results predicted by the microarray hybridizations. The PCR primers were designed to target the flanking regions of each deletion so that the amplified region spanned the missing locus. The primers used in this study are listed in Table 3. The PCRs were performed under the following conditions: $20 \mu \mathrm{l}$ total reaction volume, $10 \mu \mathrm{l}$ Hotstart (Qiagen), $10 \mathrm{pmol}$ of each primer (Eurogentec, Seraing, Belgium), $10 \mathrm{ng}$ chromosomal DNA and 5\% DMSO or 1.0 M Betaine (Sigma, St. Louis, USA). The amplification was carried out in a Geneamp PCR system 9700 thermocycler (Applied Biosystems, Foster City, USA) according manufacturer's recommendations. After amplification, 10 $\mu \mathrm{l}$ of each PCR product was observed via $1 \%$ agarose gel electrophoresis with SYBR-Safe (Molecular Probes, Carlsbad, USA) staining. To determine the deletion boundaries, PCR products were purified using ExoSAP-IT ${ }^{\circledast}$ (USB, Cleveland, USA). Subsequently the purified PCR products were sequenced using standard Big Dye Terminator v 3.1 (Applied Biosystems, Foster City, USA). Nucleotide sequencing was performed with an Applied Biosystems 3700 DNA Analyzer. Sequence data obtained from the ABI- 3700 was compared to the $B$. pertussis sequence Tohama I using the DNA-sequence analysis program KODON to determine the precise location of each deletion.

\section{Abbreviations}

CGH: Comparative Genomic Hybridization; MLST: Multi-Locus Sequence Typing; MLVA: Multiple-Locus Variable number tandem repeat Analysis.

\section{Authors' contributions}

AJK, initiated and contributed to the design of the oligonucleotides used for the microarray, setup, designed and

Table 3: Primers used in this study.

\begin{tabular}{|c|c|c|}
\hline Name & Description & Sequence (5'-3') \\
\hline BP09I0-F & & CGGGGTGGGGATGAGCAAT \\
\hline BP0934-R & & CCACGTTTTCACCCACCCAGA \\
\hline BPII35-F & & GGCCAGGTTCTCCTTGGCG \\
\hline BPII4I-R & & GCGCGTGATAGTCGGCCAG \\
\hline BPII56-F & & GTCGAACAGGGAGACCTGGTGC \\
\hline BPII78-R & & GCAGTGGAGCCCCGGTTTC \\
\hline BPI553-F & & GGATGGCGACCGCTTTCTTG \\
\hline BPI553-R & & AGCATGCCGCATTTTTCATCG \\
\hline BPI946ak-2F & & ATCATATCCCGCGCGTCCAG \\
\hline BPI969ak-2R & & GCCCGAACAGCCCAGGATC \\
\hline BPI948ak-IR & & TAGGCCCCCATGGTGGACTG \\
\hline FWBP46-69seq3 & Sequence primer & CAAATGGCTGGGCCGCTTCCTGG \\
\hline FWBP46-69seq4 & Sequence primer & TCCCCAGCGCCGTCCAGTTCCTC \\
\hline 2408 seq-F & Sequence primer & ATGACGGCGTCAAACCCACC \\
\hline 288 I seq-F & Sequence primer & GCGCGGGTGACAGATGGAG \\
\hline I858seq-F & Sequence primer & AAGCTGGGACGTATCCAGCGC \\
\hline I 554 seq-R & Sequence primer & CCAGCCATTTGCGCACAGTC \\
\hline 221 I seq-F & Sequence primer & CTTGCGTGAGTGGGCTTACGC \\
\hline BP2। $20-F 2$ & & TGGCGGAAAGCCGCTACCT \\
\hline BP2। 24-R2 & & TACGACATTCCCGGTGCCTTG \\
\hline BP2820-F2 & & TGTCCAATTCCCTGGTGCTGG \\
\hline BP2840-R & & AAAGAGGCCTTGTTCCGCGAA \\
\hline
\end{tabular}


carried out (part of) the microarray studies, participated in the MLST-, MLVA -and microarray data analysis and wrote the manuscript. TvG, carried out the MLST, MLVA and microarray experiments and commented on the manuscript. JLAP participated in the statistical analysis. HGJvdH participated in the MLST, MLVA and microarray data analysis. QH initiated and contributed to the development of the oligonucleotides used for the microarray and commented on the manuscript. DD participated in analysis of data. KH participated in the MLST analysis. MvG participated in the MLVA analysis. KvL participated in the microarray studies. FRM contributed to the conception and design of experiments and was involved in writing the manuscript. All authors read and approved the final manuscript.

\section{Additional material}

\section{Additional file 1}

Composition of MLVA types in $\mathrm{B}$. pertussis strains

Click here for file

[http://www.biomedcentral.com/content/supplementary/1471-

2164-9-311-S1.doc]

\section{Additional file 2}

Distribution of MLVA types per year

Click here for file

[http://www.biomedcentral.com/content/supplementary/1471-

2164-9-311-S2.doc]

\section{Additional file 3}

Annotation of genes missing in circulating strains, from 1993-2004, RD3

Click here for file

[http://www.biomedcentral.com/content/supplementary/14712164-9-311-S3.doc]

\section{Additional file 4}

Annotation of genes missing in circulating strains, from 1993-2004, RD5

Click here for file

[http://www.biomedcentral.com/content/supplementary/14712164-9-311-S4.doc]

\section{Additional file 5}

Annotation of genes missing in circulating strains, from 1993-2004, RD10

Click here for file

[http://www.biomedcentral.com/content/supplementary/1471-

2164-9-311-S5.doc]

\section{Additional file 6}

Annotation of genes missing in circulating strains, from 1988-1990, RD1 *

Click here for file

[http://www.biomedcentral.com/content/supplementary/14712164-9-311-S6.doc]

\author{
Additional file 7 \\ Annotation of genes missing in circulating strains, from 1993-2004, RD- \\ 6 \\ Click here for file \\ [http://www.biomedcentral.com/content/supplementary/1471- \\ 2164-9-311-S7.doc]
}

\section{Additional file 8}

Annotation of genes missing in circulating strains, from 1993-2004, RD27

Click here for file

[http://www.biomedcentral.com/content/supplementary/1471-

2164-9-311-S8.doc]

\section{Additional file 9}

Annotation of genes missing in circulating strains, from 1993-2004, RD28

Click here for file

[http://www.biomedcentral.com/content/supplementary/1471-

2164-9-311-S9.doc]

\section{Additional file 10}

Annotation of genes missing in circulating strains, from 1993-2004, RD29

Click here for file

[http://www.biomedcentral.com/content/supplementary/1471-

2164-9-311-S10.doc]

\section{Additional file 11}

Annotation of genes missing in circulating strains, from 1993-2004, RD18

Click here for file

[http://www.biomedcentral.com/content/supplementary/1471-

2164-9-311-S11.doc]

\section{Additional file 12}

B. pertussis strains used in this study

Click here for file

[http://www.biomedcentral.com/content/supplementary/1471-

2164-9-311-S12.doc]

\section{References}

I. Gueirard P, Weber C, Le Coustumier A, Guiso N: Human Bordetella bronchiseptica infection related to contact with infected animals: persistence of bacteria in host. J Clin Microbiol 1995, 33:2002-2006.

2. Diavatopoulos DA, Cummings CA, Schouls LM, Brinig MM, Relman DA, Mooi FR: Bordetella pertussis, the causative agent of whooping cough, evolved from a distinct, human-associated lineage of B. bronchiseptica. PLoS Pathog 2005, I:e45.

3. van der Zee A, Groenendijk H, Peeters M, Mooi FR: The differentiation of Bordetella parapertussis and Bordetella bronchiseptica from humans and animals as determined by DNA polymorphism mediated by two different insertion sequence elements suggests their phylogenetic relationship. Int J Syst Bacteriol 1996, 46:640-647.

4. Crowcroft NS, Pebody RG: Recent developments in pertussis. Lancet 2006, 367:1926-1936.

5. de Melker HE, Schellekens JF, Neppelenbroek SE, Mooi FR, Rumke $\mathrm{HC}$, Conyn-van Spaendonck MA: Reemergence of pertussis in the highly vaccinated population of the Netherlands: observations on surveillance data. Emerg Infect Dis 2000, 6:348-357. 
6. Guris D, Strebel PM, Bardenheier B, Brennan M, Tachdjian R, Finch E, Wharton $M$, Livengood JR: Changing epidemiology of pertussis in the United States: increasing reported incidence among adolescents and adults, 1990-1996. Clin Infect Dis 1999, 28:1230-1237.

7. Mooi FR, Van Loo IH, King AJ: Adaptation of Bordetella pertussis to vaccination: a cause for its reemergence? Emerg Infect Dis 200I, 7:526-528.

8. de Melker HE, Conyn-van Spaendonck MA, Rumke HC, van Wijngaarden JK, Mooi FR, Schellekens JF: Pertussis in The Netherlands: an outbreak despite high levels of immunization with whole-cell vaccine. Emerg Infect Dis 1997, 3:175-178.

9. Mooi FR, van Oirschot H, Heuvelman K, van der Heide HG, Gaastra W, Willems RJ: Polymorphism in the Bordetella pertussis virulence factors P.69/pertactin and pertussis toxin in The Netherlands: temporal trends and evidence for vaccine-driven evolution. Infect Immun 1998, 66:670-675.

10. Mooi FR, He Q, van Oirschot H, Mertsola J: Variation in the Bordetella pertussis virulence factors pertussis toxin and pertactin in vaccine strains and clinical isolates in Finland. Infect Immun 1999, 67:3133-3134.

II. Mastrantonio P, Spigaglia P, van Oirschot $H$, van der Heide HG, Heuvelman K, Stefanelli P, Mooi FR: Antigenic variants in Bordetella pertussis strains isolated from vaccinated and unvaccinated children. Microbiology 1999, I45 ( Pt 8):2069-2075.

12. Cassiday P, Sanden G, Heuvelman K, Mooi F, Bisgard KM, Popovic T: Polymorphism in Bordetella pertussis pertactin and pertussis toxin virulence factors in the United States, 1935-1999. J Infect Dis 2000, 182:1402-1408.

13. Van Loo IH, Mooi FR: Changes in the Dutch Bordetella pertussis population in the first $\mathbf{2 0}$ years after the introduction of whole-cell vaccines. Microbiology 2002, I 48:20 I I-20I8.

14. van Boven M, de Melker HE, Schellekens JF, Kretzschmar M: A model based evaluation of the 1996-7 pertussis epidemic in The Netherlands. Epidemiol Infect 200I, I 27:73-85.

15. Van Loo IH, Heuvelman KJ, King AJ, Mooi FR: Multilocus sequence typing of Bordetella pertussis based on surface protein genes. J Clin Microbiol 2002, 40: 1994-200I.

16. Van Amersfoorth SC, Schouls LM, van der Heide HG, Advani A, Hallander $\mathrm{HO}$, Bondeson K, Von Konig CH, Riffelmann M, Vahrenholz C, Guiso N, Caro V, Njamkepo E, He Q, Mertsola J, Mooi FR: Analysis of Bordetella pertussis populations in European countries with different vaccination policies. I Clin Microbiol 2005, 43:2837-2843.

17. Packard ER, Parton R, Coote JG, Fry NK: Sequence variation and conservation in virulence-related genes of Bordetella pertussis isolates from the UK. J Med Microbiol 2004, 53:355-365.

18. Tsang RS, Lau AK, Sill ML, Halperin SA, Van Caeseele P, Jamieson F, Martin IE: Polymorphisms of the fimbria fim 3 gene of Bordetella pertussis strains isolated in Canada. J Clin Microbiol 2004, 42:5364-5367.

19. Borisova O, Kombarova SY, Zakharova NS, van Gent M, Aleshkin VA, Mazurova I, Mooi FR: Antigenic divergence between Bordetella pertussis clinical isolates from Moscow, Russia, and vaccine strains. Clin vaccine Immunol 2007, 14:234-238.

20. Schouls LM, van der Heide HG, Vauterin L, Vauterin P, Mooi FR: Multiple-locus variable-number tandem repeat analysis of Dutch Bordetella pertussis strains reveals rapid genetic changes with clonal expansion during the late 1990s. J Bacteriol 2004, 186:5496-5505.

21. Mooi FR, van Loo IH, He Q, van Gent M, Heuvelman CJ, de Greeff SC, Diavatopoulos DA, Teunis PF, Nagelkerke NJ, Mertsola J: Bordetella Pertussis Strains With a Novel Allele for the Pertussis Toxin Promoter Are Associated With Increased Virulence and the Resurgence of Whooping Cough. 2008 in press.

22. Cummings CA, Brinig MM, Lepp PW, van de PS, Relman DA: Bordetella species are distinguished by patterns of substantial gene loss and host adaptation. J Bacteriol 2004, 1 86: | 484- I492.

23. Brinig MM, Cummings CA, Sanden GN, Stefanelli P, Lawrence A, Relman DA: Significant gene order and expression differences in Bordetella pertussis despite limited gene content variation. J Bacteriol 2006, I 88:2375-2382.

24. Caro V, Hot D, Guigon G, Hubans C, Arrive M, Soubigou G, RenauldMongenie G, Antoine R, Locht C, Lemoine Y, Guiso N: Temporal analysis of French Bordetella pertussis isolates by compara- tive whole-genome hybridization. Microbes Infect 2006, 8:2228-2235

25. Heikkinen E, Kallonen T, Saarinen L, Sara R, King AJ, Mooi FR, Soini JT, Mertsola J, He Q: Comparative Genomics of Bordetella pertussis Reveals Progressive Gene Loss in Finnish Strains. PLoS ONE 2007, 2:e904.

26. Parkhill J, Sebaihia M, Preston A, Murphy LD, Thomson N, Harris DE, Holden MT, Churcher CM, Bentley SD, Mungall KL, Cerdeno-Tarraga AM, Temple L, James K, Harris B, Quail MA, Achtman M, Atkin R, Baker S, Basham D, Bason N, Cherevach I, Chillingworth T, Collins M, Cronin A, Davis P, Doggett J, Feltwell T, Goble A, Hamlin N, Hauser $\mathrm{H}$, Holroyd S, Jagels K, Leather S, Moule S, Norberczak H, O'Neil S, Ormond D, Price C, Rabbinowitsch E, Rutter S, Sanders M, Saunders D, Seeger K, Sharp S, Simmonds M, Skelton J, Squares R, Squares S, Stevens K, Unwin L, Whitehead S, Barrell BG, Maskell DJ: Comparative analysis of the genome sequences of Bordetella pertussis, Bordetella parapertussis and Bordetella bronchiseptica. Nat Genet 2003, 35:32-40.

27. Mooi FR, Hallander H, Wirsing von Konig CH, Hoet B, Guiso N: Epidemiological typing of Bordetella pertussis isolates: recommendations for a standard methodology. Eur J Clin Microbiol Infect Dis 2000, 19:174-181.

28. Garmory HS, Titball RW: ATP-binding cassette transporters are targets for the development of antibacterial vaccines and therapies. Infect Immun 2004, 72:6757-6763.

29. Lawrence JG: Common themes in the genome strategies of pathogens. Curr Opin Genet Dev 2005, I5:584-588.

30. Operon web site 2008 [http://www.operon.com/arrays].

31. r-project 2008 [http://www.r-project.org]

32. Benjamini $Y$, Hochberg $Y$ : Controlling the False Discovery Rate: A Practical and Powerful Approach to Multiple Testing. Journal of the Royal Statistical Society Series B (Methodological) 1995, 57:289-300

33. Saeed Al, Sharov V, White J, Li J, Liang W, Bhagabati N, Braisted J, Klapa M, Currier T, Thiagarajan M, Sturn A, Snuffin M, Rezantsev A, Popov D, Ryltsov A, Kostukovich E, Borisovsky I, Liu Z, Vinsavich A, Trush V, Quackenbush J: TM4: a free, open-source system for microarray data management and analysis. Biotechniques 2003, 34:374-378

Publish with Biomed Central and every scientist can read your work free of charge

"BioMed Central will be the most significant development for disseminating the results of biomedical research in our lifetime. "

Sir Paul Nurse, Cancer Research UK

Your research papers will be:

- available free of charge to the entire biomedical community

- peer reviewed and published immediately upon acceptance

- cited in PubMed and archived on PubMed Central

- yours - you keep the copyright
BiolMedcentral 